\title{
Elastic Task Model For Adaptive Rate Control
}

\author{
Giorgio C. Buttazzo, Giuseppe Lipari, and Luca Abeni \\ Scuola Superiore S. Anna, Pisa, Italy \\ \{giorgio,lipari\}@sssup.it, luca@hartik.sssup.it
}

\begin{abstract}
An increasing number of real-time applications, related to multimedia and adaptive control systems, require greater flexibility than classical real-time theory usually permits. In this paper we present a novel periodic task model, in which tasks' periods are treated as springs, with given elastic coefficients. Under this framework, periodic tasks can intentionally change their execution rate to provide different quality of service, and the other tasks can automatically adapt their periods to keep the system underloaded. The proposed model can also be used to handle overload conditions in a more flexible way, and provide a simple and efficient mechanism for controlling the quality of service of the system as a function of the current load.
\end{abstract}

\section{Introduction}

Periodic activities represent the major computational demand in many real-time applications, since they provide a simple way to enforce timing constraints through rate control. For instance, in digital control systems, periodic tasks are associated with sensory data acquisition, low-level servoing, control loops, action planning, and system monitoring. In such applications, a necessary condition for guaranteeing the stability of the controlled system is that each periodic task is executed at a constant rate, whose value is computed at the design stage based on the characteristics of the environment and on the required performance. For critical control applications (i.e., those whose failure may cause catastrophic consequences), the feasibility of the schedule has to be guaranteed a priori and no task can change its period while the system is running.

Such a rigid framework in which periodic tasks operate is also determined by the schedulability analysis that must be performed on the task set to guarantee its feasibility under the imposed constraints. To simplify the analysis, in fact, some feasibility tests for periodic tasks are based on quite rigid assumptions. For example, in the original Liu and Layland's paper [7] on the Rate Monotonic (RM) and the
Earliest Deadline First (EDF) algorithms, a periodic task $\tau_{i}$ is modeled as a cyclical processor activity characterized by two parameters, the computation time $C_{i}$ and the period $T_{i}$, which are considered to be constant for all task instances. This is a reasonable assumption for most real-time control systems, but it can be too restrictive for other applications.

For example, in multimedia systems timing constraints can be more flexible and dynamic than control theory usually permits. Activities such as voice sampling, image acquisition, sound generation, data compression, and video playing, are performed periodically, but their execution rates are not as rigid as in control applications. Missing a deadline while displaying an MPEG video may decrease the quality of service (QoS), but does not cause critical system faults. Depending on the requested QoS, tasks may increase or decrease their execution rate to accommodate the requirements of other concurrent activities.

If a multimedia task manages compressed frames, the time for coding/decoding each frame can vary significantly, hence the worst-case execution time (WCET) of the task can be much bigger than its mean execution time. Since hard real-time tasks are guaranteed based on their WCET (and not based on mean execution times), multimedia activities can cause a waste of the CPU resource, if treated as "rigid" hard real-time tasks.

In order to provide theoretical support for such applications, some work has been done to deal with tasks with variable computation times. In [18] a probabilistic guarantee is performed on tasks whose execution times have known distribution. In [17], the authors provide an upper bound of completion times of jobs chains with variable execution times and arbitrary release times. In [9], a guarantee is computed for tasks whose jobs are characterized by variable computation times and interarrival times, occurring with a cyclical pattern. In [8], a capacity reservation technique is used to handle tasks with variable computation time and bound their computational demand.

Even in some control application, there are situations in which periodic tasks could be executed at different rates in different operating conditions. For example, in a flight control system, the sampling rate of the altimeters is a function 
of the current altitude of the aircraft: the lower the altitude, the higher the sampling frequency. A similar need arises in robotic applications in which robots have to work in unknown environments where trajectories are planned based on the current sensory information. If a robot is equipped with proximity sensors, in order to maintain a desired performance, the acquisition rate of the sensors must increase whenever the robot is approaching an obstacle.

In other situations, the possibility of varying tasks' rates increases the flexibility of the system in handling overload conditions, providing a more general admission control mechanism. For example, whenever a new task cannot be guaranteed by the system, instead of rejecting the task, the system can try to reduce the utilizations of the other tasks (by increasing their periods in a controlled fashion) to decrease the total load and accommodate the new request. Unfortunately, there is no uniform approach for dealing with these situations. For example, Kuo and Mok [4] propose a load scaling technique to gracefully degrade the workload of a system by adjusting the periods of processes. In this work, tasks are assumed to be equally important and the objective is to minimize the number of fundamental frequencies to improve schedulability under static priority assignments. In [12], Nakajima and Tezuka show how a real-time system can be used to support an adaptive application: whenever a deadline miss is detected, the period of the failed task is increased. In [13], Seto et al. change tasks' periods within a specified range to minimize a performance index defined over the task set. This approach is effective at a design stage to optimize the performance of a discrete control system, but cannot be used for on-line load adjustment. In [6], Lee, Rajkumar and Mercer propose a number of policies to dynamically adjust the tasks' rates in overload conditions. In [1], Abdelzaher, Atkins, and Shin present a model for QoS negotiation to meet both predictability and graceful degradation requirements in cases of overload. In this model, the QoS is specified as a set of negotiation options, in terms of rewards and rejection penalties. In $[10,11]$, Nakajima shows how a multimedia activity can adapt its requirements during transient overloads by scaling down its rate or its computational demand. However, it is not clear how the the QoS can be increased when the system is underloaded.

Although these approaches may lead to interesting results in specific applications, we believe that a more general framework can be used to avoid a proliferation of policies and treat different applications in a uniform fashion.

In this paper we present a novel framework, the elastic model, which has the following advantages:

- it allows tasks to intentionally change their execution rate to provide different quality of service;

- it can handle overload situations in a more flexible way;
- it provides a simple and efficient method for controlling the quality of service of the system as a function of the current load.

The rest of the paper is organized as follows. Section 2 presents the elastic task model. Section 3 illustrates the equivalence of the model with a mechanical system of linear springs. Section 4 describes the guarantee algorithm for a set of elastic tasks. Section 5 presents some theoretical results which validate the proposed model. Section 6 illustrates some experimental results achieved on the HARTIK kernel. Finally, Section 7 contains our conclusions and future work.

\section{The elastic model}

The basic idea behind the elastic model proposed in this paper is to consider each task as flexible as a spring with a given rigidity coefficient and length constraints. In particular, the utilization of a task is treated as an elastic parameter, whose value can be modified by changing the period or the computation time. To simplify the presentation of the model, in this paper we assume that the computation is fixed, while the period can be varied within a specified range.

Each task is characterized by five parameters: a computation time $C_{i}$, a nominal period $T_{i_{0}}$, a minimum period $T_{i_{\text {min }}}$, a maximum period $T_{i_{\max }}$, and an elastic coefficient $e_{i} \geq 0$, which specifies the flexibility of the task to vary its utilization for adapting the system to a new feasible rate configuration. The greater $e_{i}$, the more elastic the task. Thus, an elastic task is denoted as:

$$
\tau_{i}\left(C_{i}, T_{i_{0}}, T_{i_{m i n}}, T_{i_{m a x}}, e_{i}\right)
$$

In the following, $T_{i}$ will denote the actual period of task $\tau_{i}$, which is constrained to be in the range $\left[T_{i_{\text {min }}}, T_{i_{\text {max }}}\right]$. Any task can vary its period according to its needs within the specified range. Any variation, however, is subject to an elastic guarantee and is accepted only if there exists a feasible schedule in which all the other periods are within their range. Consider, for example, a set of three tasks, whose parameters are shown in Table 1. With the nominal periods, the task set is schedulable by EDF since

$$
U_{p}=\frac{10}{20}+\frac{10}{40}+\frac{15}{70}=0.964<1 .
$$

If task $\tau_{3}$ reduces its period to 50 , no feasible schedule exists, since the utilization would be greater than 1 :

$$
U_{p}=\frac{10}{20}+\frac{10}{40}+\frac{15}{50}=1.05>1 .
$$

However, notice that a feasible schedule exists $\left(U_{p}=0.977\right)$ for $T_{1}=22, T_{2}=45$, and $T_{3}=50$, hence the system can accept the higher request rate of $\tau_{3}$ by slightly decreasing 


\begin{tabular}{|c|c|c|c|c|c|}
\hline Task & $C_{i}$ & $T_{i_{0}}$ & $T_{i_{\min }}$ & $T_{i_{\max }}$ & $e_{i}$ \\
\hline \hline$\tau_{1}$ & 10 & 20 & 20 & 25 & 1 \\
\hline$\tau_{2}$ & 10 & 40 & 40 & 50 & 1 \\
\hline$\tau_{3}$ & 15 & 70 & 35 & 80 & 1 \\
\hline
\end{tabular}

Table 1. Task set parameters used for the example.

the rates of $\tau_{1}$ and $\tau_{2}$. Task $\tau_{3}$ can even run with a period $T_{3}=40$, since a feasible schedule exists with periods $T_{1}$ and $T_{2}$ within their range. In fact, when $T_{1}=24, T_{2}=50$, and $T_{3}=40, U_{p}=0.992$. Finally, notice that if $\tau_{3}$ requires to run with a period $T_{3}=35$, there is no feasible schedule with periods $T_{1}$ and $T_{2}$ within their range, hence the request of $\tau_{3}$ to execute with a period $T_{3}=35$ must be rejected.

Clearly, for a given value of $T_{3}$, there can be many different period configurations which lead to a feasible schedule, hence one of the possible feasible configurations must be selected. The great advantage of using an elastic model is that the policy for selecting a solution is implicitly encoded in the elastic coefficients provided by the user. Thus, each task is varied based on its current elastic status and a feasible configuration is found, if there exists one.

As another example, consider the same set of three tasks with their nominal periods, but suppose that a new periodic task $\tau_{4}(5,30,30,30,0)$ enters the system at time $t$. In a rigid scheduling framework, $\tau_{4}$ (or some other task selected by a more sophisticated rejection policy) must be rejected, because the new task set is not schedulable, being

$$
U_{p}=\sum_{i=1}^{4} \frac{C_{i}}{T_{i_{0}}}=1.131>1 .
$$

Using an elastic model, however, $\tau_{4}$ can be accepted if the periods of the other tasks can be increased in such a way that the total utilization is less than one and all the periods are within their range. In our specific example, the period configuration given by $T_{1}=23, T_{2}=50, T_{3}=80$, $T_{4}=30$, creates a total utilization $U_{p}=0.989$, hence $\tau_{4}$ can be accepted.

The elastic model also works in the other direction. Whenever a periodic task terminates or decreases its rate, all the tasks that have been previously "compressed" can increase their utilization or even return to their nominal periods, depending on the amount of released bandwidth.

It is worth to observe that the elastic model is more general than the classic Liu and Layland's task model, so it does not prevent a user from defining hard real-time tasks. In fact, a task having $T_{i_{\min }}=T_{i_{\max }}=T_{i_{0}}$ is equivalent to a hard real-time task with fixed period, independently of its elastic coefficient. A task with $e_{i}=0$ can arbitrarily vary its period within its specified range, but it cannot be varied by the system during load reconfiguration.

\section{Equivalence with a spring system}

To understand how an elastic guarantee is performed in this model, it is convenient to compare an elastic task $\tau_{i}$ with a linear spring $S_{i}$ characterized by a rigidity coefficient $k_{i}$, a nominal length $x_{i_{0}}$, a minimum length $x_{i_{\min }}$ and a maximum length $x_{i_{\max }}$. In the following, $x_{i}$ will denote the actual length of spring $S_{i}$, which is constrained to be in the range $\left[x_{i_{\text {min }}}, x_{i_{\text {max }}}\right]$.

In this comparison, the length $x_{i}$ of the spring is equivalent to the task's utilization factor $U_{i}=C_{i} / T_{i}$, and the rigidity coefficient $k_{i}$ is equivalent to the inverse of task's elasticity $\left(k_{i}=1 / e_{i}\right)$. Hence, a set of $n$ tasks with total utilization factor $U_{p}=\sum_{i=1}^{n} U_{i}$ can be viewed as a sequence of $n$ springs with total length $L=\sum_{i=1}^{n} x_{i}$.

Using the same notation introduced by Liu and Layland [7], let $U_{l u b}^{A}$ be the least upper bound of the total utilization factor for a given scheduling algorithm $A$ (we recall that for $n$ tasks $U_{l u b}^{R M}=n\left(2^{1 / n}-1\right)$ and $\left.U_{l u b}^{E D F}=1\right)$. Hence, a task set can be schedulable by $A$ if $U_{p} \leq U_{\text {lub }}^{A}$. Under EDF, such a schedulability condition becomes necessary and sufficient.

Under the elastic model, given a scheduling algorithm $A$ and a set of $n$ tasks with $U_{p}>U_{\text {lub }}^{A}$, the objective of the guarantee is to compress tasks' utilization factors in order to achieve a new utilization $U_{p}^{\prime} \leq U_{l u b}^{A}$ such that all the periods are within their ranges. In the linear spring system, this is equivalent of compressing the springs so that the new total length $L^{\prime}$ is less than or equal to a given maximum length $L_{\text {max }}$. More formally, in the spring system the problem can be stated as follows.

Given a set of $n$ springs with known rigidity and length constraints, if $L>L_{\max }$, find a set of new lengths $x_{i}^{\prime}$ such that $x_{i}^{\prime} \in\left[x_{i_{\text {min }}}, x_{i_{\text {max }}}\right]$ and $L^{\prime}=L_{d}$, where $L_{d}$ is any arbitrary desired length such that $L_{d}<L_{\max }$.

For the sake of clarity, we first solve the problem for a spring system without length constraints, then we show how the solution can be modified by introducing length constraints, and finally we show how the solution can be adapted to the case of a task set.

\subsection{Springs with no length constraints}

Consider a set $\Gamma$ of $n$ springs with nominal length $x_{i_{0}}$ and rigidity coefficient $k_{i}$ positioned one after the other, as depicted in Figure 1. Let $L_{0}$ be the total length of the array, that is the sum of the nominal lengths: $L_{0}=\sum_{i=1}^{n} x_{i_{0}}$. If 

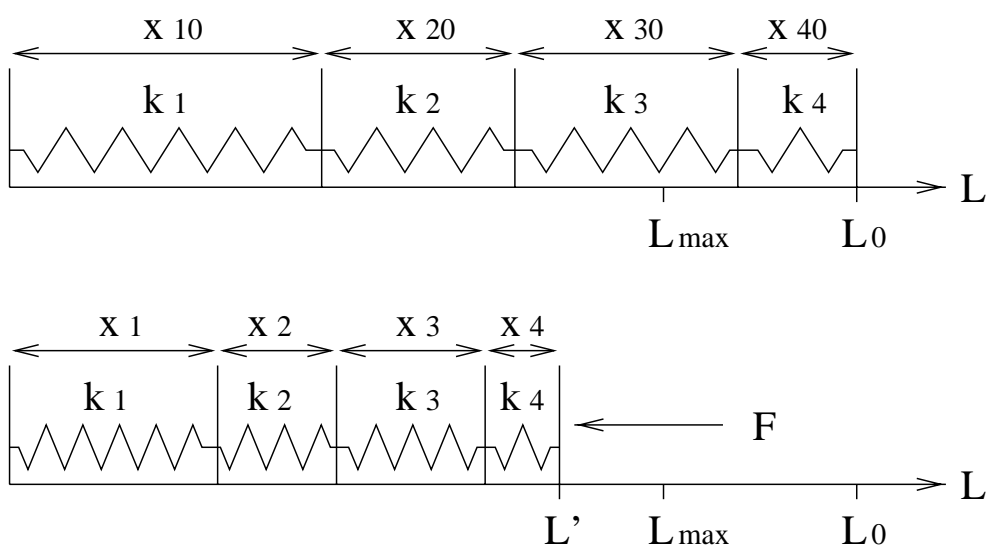

Figure 1. A linear spring system: the total length is $L_{0}$ when springs are uncompressed (a); and $L<L_{0}$ when springs are compressed (b).

the array is compressed so that its total length is equal to a desired length $L_{d}\left(0<L_{d}<L_{0}\right)$, the first problem we want to solve is to find the new length $x_{i}$ of each spring, assuming that for all $i, 0<x_{i}<x_{i_{0}}$. Being $L_{d}$ the total length of the compressed array of springs, we have:

$$
L_{d}=\sum_{i=1}^{n} x_{i} .
$$

If $F$ is the force that keeps a spring in its compressed state, then, for the equilibrium of the system, it must be:

$$
\forall i \quad F=k_{i}\left(x_{i_{0}}-x_{i}\right) .
$$

Solving the equations (1) and (2) for the unknown $x_{1}, x_{2}$, $\ldots, x_{n}$, we have:

$$
\forall i \quad x_{i}=x_{i_{0}}-\left(L_{0}-L_{d}\right) \frac{K / /}{k_{i}}
$$

where

$$
K_{/ /}=\frac{1}{\sum_{i=1}^{n} \frac{1}{k_{i}}} .
$$

\subsection{Introducing length constraints}

If each spring has a length constraint, in the sense that its length cannot be less than a minimum value $x_{i_{m i n}}$, then the problem of finding the values $x_{i}$ requires an iterative solution. In fact, if during compression one or more springs reach their minimum length, the additional compression force will only deform the remaining springs. Thus, at each instant, the set $\Gamma$ can be divided into two subsets: a set $\Gamma_{f}$ of fixed springs having minimum length, and a set $\Gamma_{v}$ of variable springs that can still be compressed. Applying equations (3) and (4) to the set $\Gamma_{v}$ of variable springs, we have

$$
\forall S_{i} \in \Gamma_{v} \quad x_{i}=x_{i_{0}}-\left(L_{0}-L_{d}+L_{f}\right) \frac{K_{v}}{k_{i}}
$$

where

$$
\begin{aligned}
L_{f} & =\sum_{S_{i} \in \Gamma_{f}} x_{i_{\text {min }}} \\
K_{v} & =\frac{1}{\sum_{S_{i} \in \Gamma_{v}} \frac{1}{k_{i}}} .
\end{aligned}
$$

Whenever there exists some spring for which equation (5) gives $x_{i}<x_{i_{m i n}}$, the length of that spring has to be fixed at its minimum value, sets $\Gamma_{f}$ and $\Gamma_{v}$ must be updated, and equations (5), (6) and (7) recomputed for the new set $\Gamma_{v}$. If there exists a feasible solution, that is, if the desired final length $L_{d}$ is greater than or equal to the minimum possible length of the array $L_{\min }=\sum_{i=1}^{n} x_{i_{\min }}$, the iterative process ends when each value computed by equations (5) is greater than or equal to its corresponding minimum $x_{i_{m i n}}$. The complete algorithm for compressing a set $\Gamma$ of $n$ springs with length constraints up to a desired length $L_{d}$ is shown in Figure 2.

\section{Compressing tasks' utilizations}

When dealing with a set of elastic tasks, equations (5), (6) and (7) can be rewritten by substituting all length parameters with the corresponding utilization factors, and the rigidity coefficients $k_{i}$ and $K_{v}$ with the corresponding elastic coefficients $e_{i}$ and $E_{v}$. Similarly, at each instant, the set $\Gamma$ of periodic tasks can be divided into two subsets: a set $\Gamma_{f}$ of fixed tasks having minimum utilization, and a set $\Gamma_{v}$ of variable tasks that can still be compressed. If $U_{i_{0}}=C_{i} / T_{i_{0}}$ 


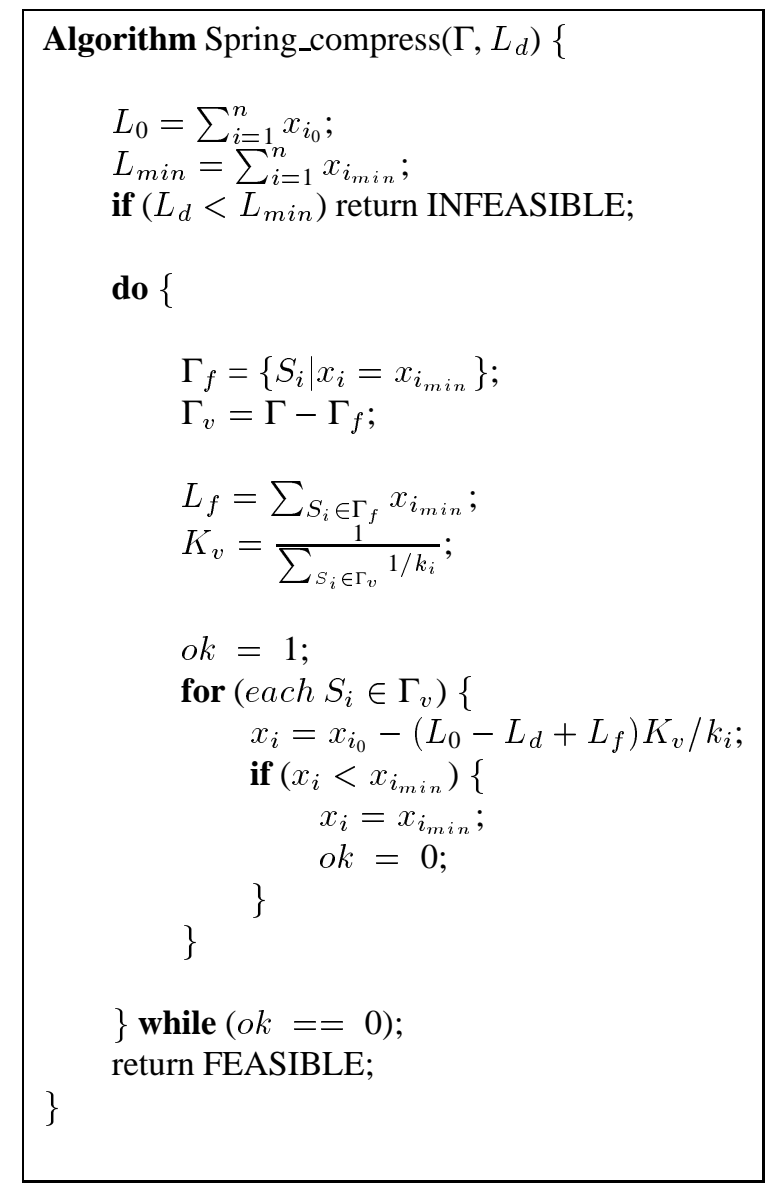

Figure 2. Algorithm for compressing a set of springs with length constraints.

is the nominal utilization of task $\tau_{i}, U_{0}$ is the sum of all the nominal utilizations, and $U_{f}$ is the total utilization factor of tasks in $\Gamma_{f}$, then to achieve a desired utilization $U_{d}<U_{0}$ each task has to be compressed up to the following utilization:

$$
\forall \tau_{i} \in \Gamma_{v} \quad U_{i}=U_{i_{0}}-\left(U_{0}-U_{d}+U_{f}\right) \frac{e_{i}}{E_{v}}
$$

where

$$
\begin{gathered}
U_{f}=\sum_{\tau_{i} \in \Gamma_{f}} U_{i_{m i n}} \\
E_{v}=\sum_{\tau_{i} \in \Gamma_{v}} e_{i} .
\end{gathered}
$$

If there exist tasks for which $U_{i}<U_{i_{m i n}}$, then the period of those tasks has to be fixed at its maximum value $T_{i_{\max }}$ (so that $U_{i}=U_{i_{\text {min }}}$ ), sets $\Gamma_{f}$ and $\Gamma_{v}$ must be updated (hence, $U_{f}$ and $E_{v}$ recomputed), and equation (8) applied

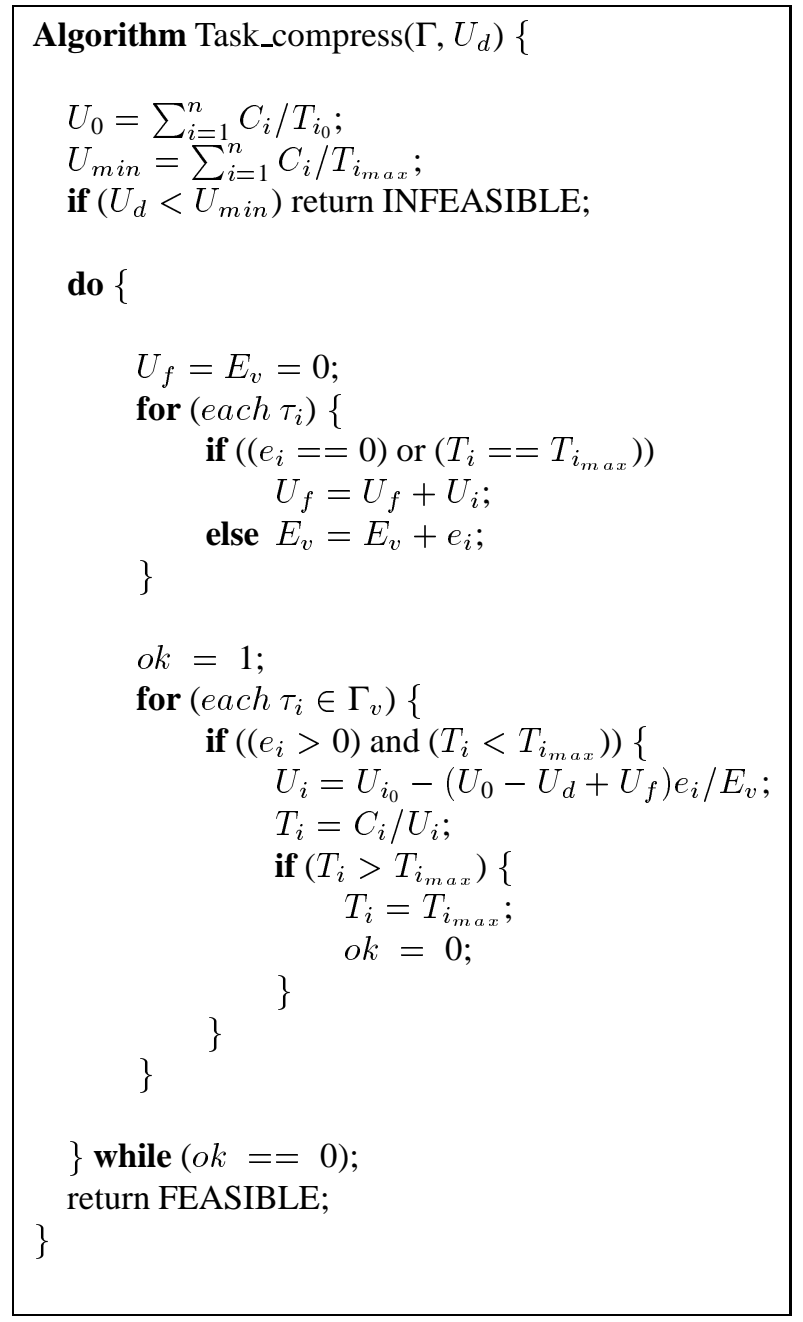

Figure 3. Algorithm for compressing a set of elastic tasks.

again to the tasks in $\Gamma_{v}$. If there exists a feasible solution, that is, if the desired utilization $U_{d}$ is greater than or equal to the minimum possible utilization $U_{\min }=\sum_{i=1}^{n} \frac{C_{i}}{T_{i_{\max }}}$, the iterative process ends when each value computed by equation (8) is greater than or equal to its corresponding minimum $U_{i_{\text {min }}}$. The algorithm ${ }^{1}$ for compressing a set $\Gamma$ of $n$ elastic tasks up to a desired utilization $U_{d}$ is shown in Figure 3.

\subsection{Decompression}

All tasks' utilizations that have been compressed to cope with an overload situation can return toward their nominal

\footnotetext{
${ }^{1}$ The actual implementation of the algorithm contains more checks on tasks' variables, which are not shown here to simplify its description.
} 
values when the overload is over. Let $\Gamma_{c}$ be the subset of compressed tasks (that is, the set of tasks with $T_{i}>T_{i_{0}}$ ), let $\Gamma_{a}$ be the set of remaining tasks in $\Gamma$ (that is, the set of tasks with $T_{i} \leq T_{i_{0}}$ ), and let $U_{d}$ be the current processor utilization of $\Gamma$. Whenever a task in $\Gamma_{a}$ decreases its rate or returns to its nominal period, all tasks in $\Gamma_{c}$ can expand their utilizations according to their elastic coefficients, so that the processor utilization is kept at the value of $U_{d}$.

Now, let $U_{c}$ be the total utilization of $\Gamma_{c}$, let $U_{a}$ be the total utilization of $\Gamma_{a}$, and let $U_{c_{0}}$ be the total utilization of tasks in $\Gamma_{c}$ at their nominal periods. It can easily be seen that if $U_{c_{0}}+U_{a} \leq U_{l u b}$ all tasks in $\Gamma_{c}$ can return to their nominal periods. On the other hand, if $U_{c_{0}}+U_{a}>U_{l u b}$, then the release operation of the tasks in $\Gamma_{c}$ can be viewed as a compression, where $\Gamma_{f}=\Gamma_{a}$ and $\Gamma_{v}=\Gamma_{c}$. Hence, it can still be performed by using equations (8), (9) and (10) and the algorithm presented in Figure 3.

\section{Theoretical validation of the model}

In this section we derive some theoretical results which validate the elastic guarantee algorithm that can be performed with this method. In particular, we show that if tasks' periods are changed at opportune instants the task set remains schedulable and no deadline is missed. The following lemmas state two properties of the EDF algorithm that are useful for proving the main theorem.

Lemma 1 In any feasible EDF schedule $\sigma$, the following condition holds:

$$
\forall t>0 \quad \sum_{i=1}^{n} \frac{\gamma_{i}(t)}{t} \geq U_{p}
$$

where $U_{p}=\sum_{i=1}^{n} C_{i} / T_{i}$ and $\gamma_{i}(t)$ is the cumulative time executed by all the instances of task $\tau_{i}$ up to $t$.

\section{Proof.}

If $\sigma(t)=I D L E$, we have that

$$
f(t)=\frac{\sum_{i=1}^{n} \gamma_{i}(t)}{t}=\frac{\sum_{i=1}^{n}\left\lceil\frac{t}{T_{i}}\right\rceil C_{i}}{t} \geq \frac{t \sum_{i=1}^{n} \frac{C_{i}}{T_{i}}}{t}=U_{p} .
$$

If $\sigma(t) \neq I D L E$ :

$$
\begin{aligned}
f(t+1) & =\frac{\sum_{i=1}^{n} \gamma_{i}(t+1)}{t+1}=\frac{\sum_{i=1}^{n} \gamma_{i}(t)+1}{t+1}= \\
& =\frac{f(t) t+1}{t+1}=f(t)+\frac{1-f(t)}{t+1} \geq f(t) .
\end{aligned}
$$

Moreover, being $\sigma(0) \neq I D L E$ and $f(1)=1 \geq U_{p}$ (because the system is feasible), we have that, for all $t>0$, $f(t) \geq U_{p}$.
Lemma 2 In any feasible EDF schedule $\sigma$, the following condition holds:

$$
\forall t>0 \quad \sum_{i=1}^{n} c_{i}(t) \leq \sum_{i=1}^{n}\left[\nu_{i}(t)-t\right] U_{i}
$$

where $U_{i}=C_{i} / T_{i}, c_{i}(t)$ is the remaining execution time of the current instance of task $\tau_{i}$ at time $t$, and $\nu_{i}(t)$ is the next release time of $\tau_{i}$ greater than or equal to $t$.

Proof.

By definition of $c_{i}(t)$, we have

$$
\begin{aligned}
c_{i}(t) & =\left\lceil\frac{t}{T_{i}}\right\rceil C_{i}-\gamma_{i}(t) \\
\nu_{i}(t) & =\left\lceil\frac{t}{T_{i}}\right\rceil T_{i}
\end{aligned}
$$

and, by Lemma 1,

$$
\begin{aligned}
\sum_{i=1}^{n} c_{i}(t) & =\sum_{i=1}^{n}\left(\left\lceil\frac{t}{T_{i}}\right] C_{i}-\gamma_{i}(t)\right) \leq \\
& \leq \sum_{i=1}^{n}\left(\left\lceil\frac{t}{T_{i}}\right] C_{i}-t U_{i}\right)= \\
& =\sum_{i=1}^{n}\left(T_{i}\left\lceil\frac{t}{T_{i}}\right]-t\right) U_{i}= \\
& =\sum_{i=1}^{n}\left(\nu_{i}(t)-t\right) U_{i} .
\end{aligned}
$$

The following theorem states a property of decompression: if at time $t$ all the periods are increased from $T_{i}$ to $T_{i}^{\prime}$, then the total utilization factor is decreased from $U_{p}$ to $U_{p}^{\prime}=\sum_{i=1}^{n} \frac{C_{i}}{T_{i}^{\prime}}$.

Theorem 1 Given a feasible task set $\Gamma$, with total utilization factor $U_{p}=\sum_{i=1}^{n} \frac{C_{i}}{T_{i}} \leq 1$, if at time $t$ all the periods are increased from $T_{i}$ to $T_{i}^{\prime} \geq T_{i}$, then for all $L>0$,

$$
D(t, t+L) \leq L U_{p}^{\prime}
$$

where $D\left(t_{1}, t_{2}\right)$ is the total processor demand of $\Gamma$ in $\left[t_{1}, t_{2}\right]$, and $U_{p}^{\prime}=\sum_{i=1}^{n} \frac{C_{i}}{T_{i}^{\prime}}$.

\section{Proof.}

As task periods are increased at time $t$, the new release time of task $\tau_{i}$ is:

$$
\nu_{i}^{\prime}(t)=\nu_{i}(t)-T_{i}+T_{i}^{\prime}
$$




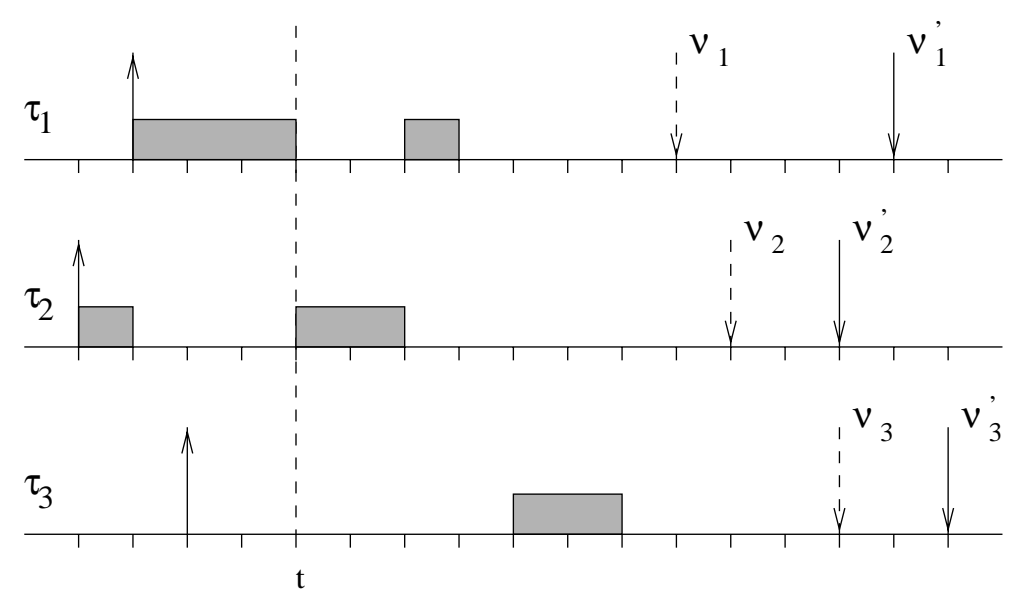

Figure 4. Example of $n$ tasks that simultaneously increase their periods at time $t$.

The total demand in $[t, t+L]$ is given by the total execution time of the instances released after or at $t$ with deadlines less than or equal to $t+L$, plus the remaining execution times of the current active instances. The situation considered in the proof is illustrated in Figure 4.

Using the result of Lemma 2 we can write:

$$
\begin{aligned}
& D(t, t+L)=\sum_{i=1}^{n}\left\lfloor\frac{t+L-\nu_{i}^{\prime}(t)}{T_{i}^{\prime}}\right\rfloor C_{i}+\sum_{i=1}^{n} c_{i}(t) \leq \\
& \leq \sum_{i=1}^{n}\left\lfloor\frac{t+L-\nu_{i}^{\prime}(t)}{T_{i}^{\prime}}\right\rfloor C_{i}+\sum_{i=1}^{n}\left[\nu_{i}(t)-t\right] U_{i} \leq \\
& \leq \sum_{i=1}^{n}\left[t+L-\nu_{i}^{\prime}(t)\right] U_{i}^{\prime}+\sum_{i=1}^{n}\left[\nu_{i}(t)-t\right] U_{i}= \\
& =\sum_{i=1} L U_{i}^{\prime}+\sum_{i=1}^{n}\left[\nu_{i}(t) U_{i}-\nu_{i}^{\prime}(t) U_{i}^{\prime}-t U_{i}+t U_{i}^{\prime}\right]= \\
& =L U_{p}^{\prime}+A .
\end{aligned}
$$

Now, we show that $A \leq 0$.

$$
\begin{aligned}
A & =\sum_{i=1}^{n}\left[\nu_{i}(t) U_{i}-\nu_{i}^{\prime}(t) \frac{T_{i}}{T_{i}^{\prime}} U_{i}-t U_{i}+t U_{i} \frac{T_{i}}{T_{i}^{\prime}}\right]= \\
& =\sum_{i=1}^{n} U_{i}\left[\nu_{i}^{\prime}(t)-T_{i}^{\prime}+T_{i}-\nu_{i}^{\prime}(t) \frac{T_{i}}{T_{i}^{\prime}}-t\left(1-\frac{T_{i}}{T_{i}^{\prime}}\right)\right] \\
& =\sum_{i=1}^{n} U_{i}\left[\nu_{i}^{\prime}(t)-t-T_{i}^{\prime}\right]\left(1-\frac{T_{i}}{T_{i}^{\prime}}\right) \leq 0
\end{aligned}
$$

Hence,

$$
D(t, t+L) \leq L U_{p}^{\prime}
$$

Notice that the property stated by Theorem 1 does not hold in case of compression. This can be seen in the example illustrated in Figure 5, where two tasks, $\tau_{1}$ and $\tau_{2}$, with computation times 3 and 2 , and periods 10 and 3 , start at time 0 . The processor utilization is $U_{p}=\frac{29}{30}$, thus the task set is schedulable by EDF. At time $t=14, \tau_{1}$ changes its period from $T_{1}=10$ to $T_{1}^{\prime}=5$. As a consequence, to keep the system schedulable, the period of $\tau_{2}$ is increased from $T_{2}=3$ to $T_{2}^{\prime}=6$. The new processor utilization is $U_{p}^{\prime}=\frac{28}{30}$, so the task set is still feasible; but, if we change the periods immediately, task $\tau_{1}$ misses its deadline at time $t=15$.

In other words, the period of a compressed task can be decreased only at its next release time. Thus, when the QoS manager receives a request of period variation, it calculates the new periods according to the elastic model: if the new configuration is found to be feasible (i.e., $U_{p}^{\prime}<1$ ), then it increases the periods of the decompressed tasks immediately, but decreases the periods of the compressed tasks only at their next release time. Theorem 1 ensures that the total processor demand in any interval $[t, t+L]$ will never exceed $L U_{p}$ and no deadline will be missed.

\section{Experimental results}

The elastic task model has been implemented on top of the HARTIK kernel [2, 5], to perform some experiments on multimedia applications and verify the results predicted by the theory. In particular, the elastic guarantee mechanism has been implemented as a high priority task, the QoS manager, activated by the other tasks when they are created or when they want to change their period. Whenever activated, the QoS manager calculates the new periods and changes them atomically. According to the result of Theorem 1, periods are changed at the next release time of the task whose period is decreased. If more tasks ask to decrease their period, the QoS manager will change them, if possible, at their next release time. 


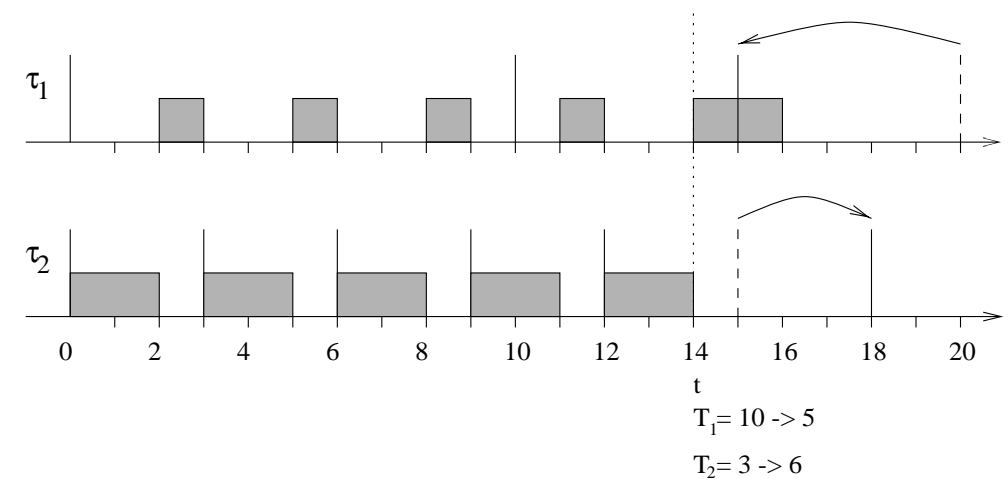

Figure 5. A task can miss its deadline if a period is decreased at arbitrary time.

\begin{tabular}{|c|c|c|c|c|c|}
\hline Task & $C_{i}$ & $T_{i_{0}}$ & $T_{i_{\min }}$ & $T_{i_{\max }}$ & $e_{i}$ \\
\hline \hline$\tau_{1}$ & 24 & 100 & 30 & 500 & 1 \\
\hline$\tau_{2}$ & 24 & 100 & 30 & 500 & 1 \\
\hline$\tau_{3}$ & 24 & 100 & 30 & 500 & 1.5 \\
\hline$\tau_{4}$ & 24 & 100 & 30 & 500 & 2 \\
\hline
\end{tabular}

\begin{tabular}{|c|r|r|r|r|r|}
\hline Task & $C_{i}$ & $T_{i_{0}}$ & $T_{i_{\min }}$ & $T_{i_{\max }}$ & $e_{i}$ \\
\hline \hline$\tau_{1}$ & 30 & 100 & 30 & 500 & 1 \\
\hline$\tau_{2}$ & 60 & 200 & 30 & 500 & 1 \\
\hline$\tau_{3}$ & 90 & 300 & 30 & 500 & 1 \\
\hline$\tau_{4}$ & 24 & 50 & 30 & 500 & 1 \\
\hline
\end{tabular}

\section{Table 2. Task set parameters used for the first experiment. Periods and computation times are expressed in milliseconds.}

In the first experiment, four periodic tasks are created at time $t=0$. Tasks' parameters are shown in Table 2, while the actual number of instances executed by each task as a function of time is shown in Figure 6. All the tasks start executing at their nominal period and, at time $t_{1}=10 \mathrm{sec}$, $\tau_{1}$ decreases its period to $T_{1}^{\prime}=33 \mathrm{msec}$. We recall that a task cannot decrease its period by itself, but must perform a request to the QoS manager, that checks the feasibility of the request and calculates the new periods for all the tasks in the system. So, at time $t_{1}$, since the schedule is found to be feasible, the period of $\tau_{1}$ is decreased and the periods of $\tau_{2}$, $\tau_{3}$ and $\tau_{4}$ are increased according to their elastic coefficients. The values of all the periods are indicated in the graph.

At time $t_{2}=20 \mathrm{sec}, \tau_{1}$ returns to its nominal period, so the QoS manager can change the periods of the other tasks to their initial values, as shown in the graph. In this manner, the QoS manager ensures that when a task requires to change its period, the task set remains schedulable and the variation of each task period can be controlled by the elastic factor.

In the second experiment, we tested the elastic model as an admission control policy. Three tasks start executing at time $t=0$ at their nominal period, while a fourth task starts at time $t_{1}=10 \mathrm{sec}$. Tasks' parameters are shown in Table 3. When $\tau_{4}$ is started, the task set is not schedulable with the current periods, thus the QoS manager, in order to accommodate the request of $\tau_{4}$, increases the periods of the other tasks according to the elastic model. The actual execution rates of the tasks are shown in Figure 7. Notice that, although the first three tasks have the same elastic coefficients, their periods are changed by a different amount, because tasks have different utilization factors.

\section{Conclusions}

In this paper we presented a flexible scheduling theory, in which periodic tasks are treated as springs, with given elastic coefficients. Under this framework, periodic tasks can intentionally change their execution rate to provide different quality of service, and the other tasks can automatically adapt their periods to keep the system underloaded. The proposed model can also be used to handle overload situations in a more flexible way. In fact, whenever a new task cannot be guaranteed by the system, instead of rejecting the task, the system can try to reduce the utilizations of the other tasks (by increasing their periods in a controlled fashion) to decrease the total load and accommodate the new request. As soon as a transient overload condition is over (because 


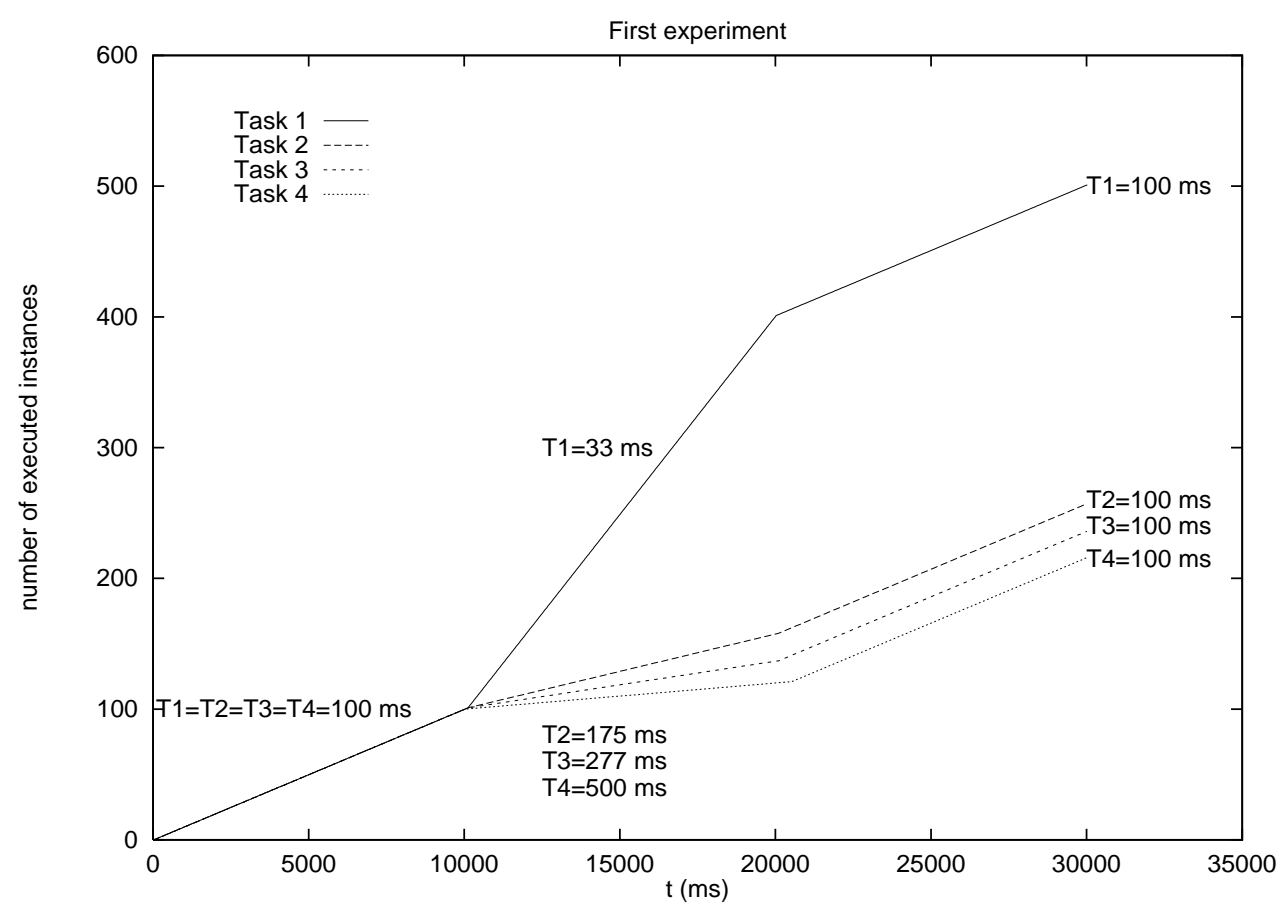

Figure 6. Dynamic period change.

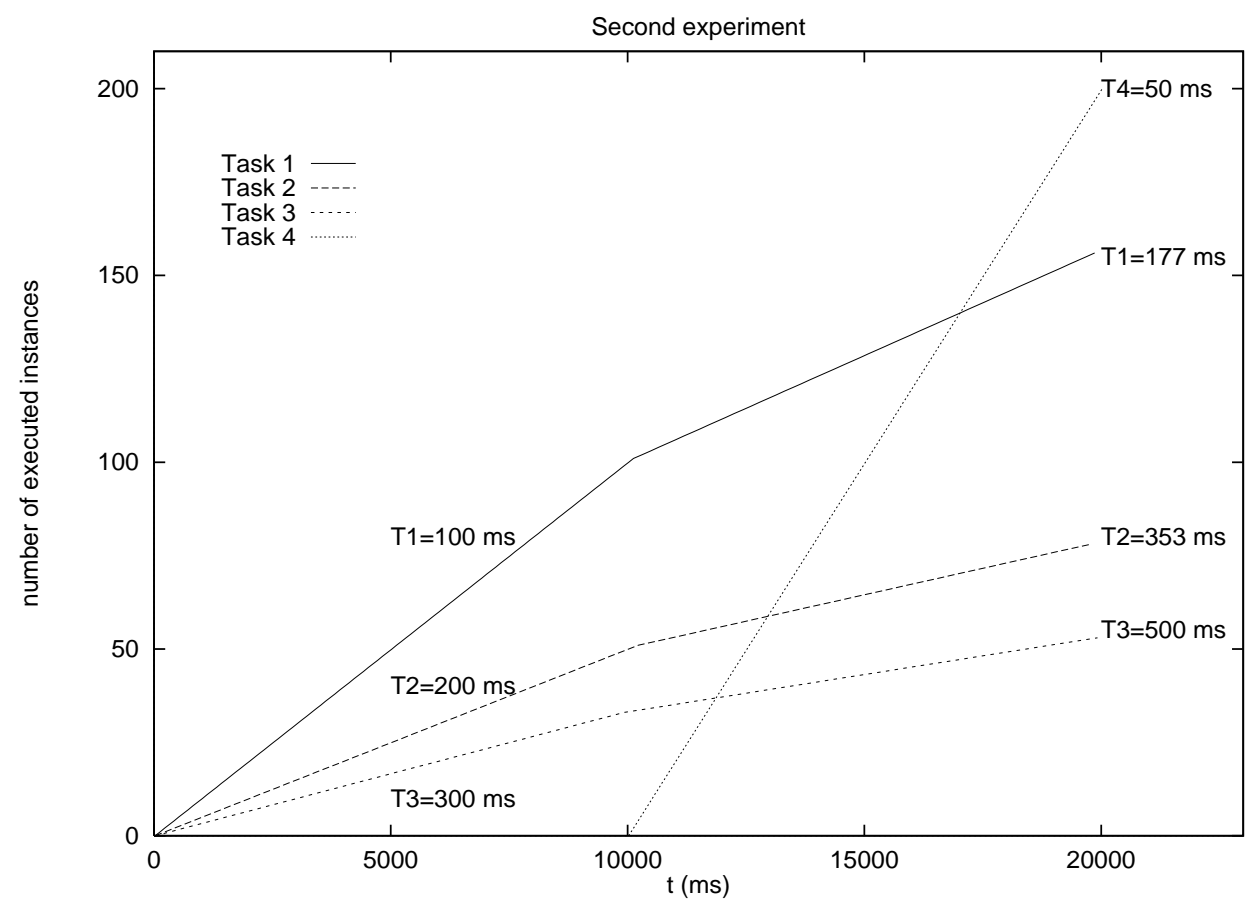

Figure 7. Dynamic task activation. 
a task terminates or voluntarily increases its period) all the compressed tasks may expand up to their original utilization, eventually recovering their nominal periods.

The major advantage of the proposed method is that the policy for selecting a solution is implicitly encoded in the elastic coefficients provided by the user. Each task is varied based on its current elastic status and a feasible configuration is found, if there exists one.

The elastic model is extremely useful for supporting both multimedia systems and control applications, in which the execution rates of some computational activities have to be dynamically tuned as a function of the current system state. Furthermore, the elastic mechanism can easily be implemented on top of classical real-time kernels, and can be used under fixed or dynamic priority scheduling algorithms. The experimental results shown in this paper have been conducted by implementing the elastic mechanism on the HARTIK kernel [2, 5].

As a future work, we are investigating the possibility of extending this method for dealing with tasks having deadlines less than periods, variable execution time, and subject to resource constraints.

\section{References}

[1] T.F. Abdelzaher, E.M. Atkins, and K.G. Shin, "QoS Negotiation in Real-Time Systems and Its Applications to Automated Flight Control," Proceedings of the IEEE Real-Time Technology and Applications Symposium, Montreal, Canada, June 1997.

[2] G. Buttazzo, "HARTIK: A Real-Time Kernel for Robotics Applications", Proceedings of the 14th IEEE Real-Time Systems Symposium, Raleigh-Durham, pp. 201-205, December 1993.

[3] M.L. Dertouzos, "Control Robotics: the Procedural Control of Physical Processes," Information Processing, 74, North-Holland, Publishing Company, 1974.

[4] T.-W. Kuo and A. K, Mok, "Load Adjustment in Adaptive Real-Time Systems," Proceedings of the 12th IEEE Real-Time Systems Symposium, December 1991.

[5] G. Lamastra, G. Lipari, G. Buttazzo, A. Casile, and F. Conticelli, "HARTIK 3.0: A Portable System for Developing Real-Time Applications," Proceedings of the IEEE Real-Time Computing Systems and Applications, Taipei, Taiwan, October 1997.

[6] C. Lee, R. Rajkumar, and C. Mercer, "Experiences with Processor Reservation and Dynamic QOS in RealTime Mach," Proceedings of Multimedia Japan 96, April 1996.
[7] C.L. Liu and J.W. Layland, "Scheduling Algorithms for Multiprogramming in a Hard real-Time Environment," Journal of the ACM 20(1), 1973, pp. 40-61.

[8] C. W. Mercer, S. Savage, and H. Tokuda, "Processor Capacity Reserves for Multimedia Operating Systems" Proceedings of the IEEE International Conference on Multimedia Computing and Systems, May 1994.

[9] A. K. Mok and D. Chen, "A multiframe model for realtime tasks," Proceedings of IEEE Real-Time System Symposium, Washington, December 1996.

[10] T. Nakajima, "Dynamic QOS Control and Resource Reservation,' IEICE, RTP'98, 1998.

[11] T. Nakajima, "Resource Reservation for Adaptive QOS Mapping in Real-Time Mach," Sixth International Workshop on Parallel and Distributed RealTime Systems, April 1998.

[12] T. Nakajima and H. Tezuka, "A Continuous Media Application supporting Dynamic QOS Control on RealTime Mach," Proceedings of the ACM Multimedia '94, 1994.

[13] D. Seto, J.P. Lehoczky, L. Sha, and K.G. Shin, “On Task Schedulability in Real-Time Control Systems," Proceedings of the IEEE Real-Time Systems Symposium, December 1997.

[14] M. Spuri, and G.C. Buttazzo, "Efficient Aperiodic Service under Earliest Deadline Scheduling", Proceedings of IEEE Real-Time System Symposium, San Juan, Portorico, December 1994.

[15] M. Spuri, G.C. Buttazzo, and F. Sensini, "Robust Aperiodic Scheduling under Dynamic Priority Systems", Proceedings of the IEEE Real-Time Systems Symposium, Pisa, Italy, December 1995.

[16] M. Spuri and G.C. Buttazzo, "Scheduling Aperiodic Tasks in Dynamic Priority Systems," Real-Time Systems, 10(2), 1996.

[17] J. Sun and J.W.S. Liu, "Bounding Completion Times of Jobs with Arbitrary Release Times and Variable Execution Times", Proceedings of IEEE Real-Time System Symposium, December 1996.

[18] T.-S. Tia, Z. Deng, M. Shankar, M. Storch, J. Sun, L.-C. Wu, and J.W.-S. Liu, "Probabilistic Performance Guarantee for Real-Time Tasks with Varying Computation Times," Proceedings of IEEE Real-Time Technology and Applications Symposium, Chicago, Illinois, January 1995. 